ISSN: 2162-3104 Print/ ISSN: 2166-3750 Online

Volume 9, Issue 2 (2019), pp. 511-534

doi: 10.32674/jis.v9i2.964

(C) Journal of International Students

https://ojed.org/jis/

\title{
Cultural Intelligence, Age and Prior Travel Experience as Predictors of Acculturative Stress and Depression among International Students Studying in China
}

\author{
Werede Tareke Gebregergis \\ Fei Huang \\ Jiangzhong Hong \\ Central China Normal University, China
}

\begin{abstract}
Mental health problems commonly prevail among international students as a result of acculturative difficulties. In light of this, the study attempted to determine the role of cultural intelligence, age and prior travel experience on acculturative stress and depression and also whether acculturative stress mediated the cultural intelligence-depression relationship. 506 international university students studying in China completed a battery of tests assessing their cultural intelligence, acculturative stress and depression. Cultural intelligence showed significantly negative correlations with both acculturative stress and depression. Students' prior travel experiences and age also significantly correlated with both acculturative stress and depression. Acculturative stress mediated the relationship between cultural intelligence and depression. Implication, limitations and future study directions were discussed as well.
\end{abstract}

Keywords: acculturative stress, cultural intelligence, depression, international students 


\section{INTRODUCTION}

Educational international exchange programs provide many advantages for international students and the host community. For instance, being part of the international intellectual learning environment community offers a tremendous opportunity for students to capitalize on their personal and professional potentials. It also provides the chance to familiarize with a wide range of cultures and thereby enriches their socio-cultural awareness and competence (Bevis, 2002). On the other hand, the full range of multidisciplinary skills and knowledge of the international students significantly enhance the intellectual capital and workforce and thereby the knowledge-based economic development of the host country (Bista, 2019). However, acclimating to the new environment of the host country may also be taxing for international students because of the accumulated pressure related to a new language, cultural values, and social norms, and the pressure may subsequently increase their feelings of stress or fear (Mustaffa $\&$ Ilias, 2013). International students were found to be at a high risk of undergoing challenges because of the acculturation related demands to the new social and educational environments (Mori, 2000; Yeh \& Inose, 2003). It is for this reason that international students are commonly found to be more stressed and depressed than the domestic students (Krämer, PröferKrämer, Stock, \& Tshiananga, 2004).

The concern about mental health problems among international students inspired many researchers to conduct studies about numerous psychological resources and personal factors that influence the students' acculturative outcomes (Brunsting, Zachry, \& Takeuchi, 2018). As part of this concern, the present study in its part has made an effort towards an accurate and comprehensive understanding of the relationship between psychological attributes as predictors and acculturative outcomes. More specifically, the study tried to examine how cultural intelligence as a psychological resource and personal characteristics like age and prior travel experience contribute to international students' psychological acculturation process and outcomes in the host environment. 


\section{THEORETICAL CONCEPTUALIZATION AND EMPIRICAL STUDIES}

\section{Acculturative Stress}

According to Berry (2005), when individuals travel to a new environment, they undergo the process of acculturation. Acculturation takes place when people experience several psychological changes associated with the 'first hand' contact in the multicultural setting (Berry, 2005). As the acculturation process has its challenges, the acculturation outcome is not always positive. Berry (1997) has conceptualized the acculturation outcomes in two ways.

First, individuals encounter easily manageable behavioral changes which are barely challenging. This process of acculturative outcome entails three processes: culture shedding, culture learning, and culture conflict. In culture shedding and culture learning, individuals make intentional, selective and purposeful alterations in their behaviors and replace them with behaviors that appropriately fit within mainstream society. These processes are mostly termed as adjustments for the reason that all the adaptive changes are more of the acculturating individual than the host community (Ward, Bochner, \& Furnham, 2005). Because of the non-problematic nature of such stages, individuals are less likely to experience significant acculturative difficulties. However, individuals may face some cultural conflicts which they may tackle through their conformity to the mainstream culture. For the individuals who search for integration, cultural conflict may be tackled in the context of multiculturalism. On the other hand, individuals who remain with sustained separation and marginalization may be sidelined from the arena of acculturation and face greater cultural conflict.

Second, acculturative stress is the appropriate conceptualization under which individuals come to realize the experience of acculturative difficulties as a result of acculturation that may not be easily resolved through normal intercultural adjustment. Given the general stress and adaptation paradigm, this approach advocates the study of the process of how individuals come to cope with problems at the initial stage and overtime. In this context, acculturative stress is an individuals' stress reaction towards the life dealings that are embedded in the involvement of acculturation (Berry, 2005) which may detrimentally affect his or her psychological well-being, causing physical and mental illness. Hence, the second conceptualization of acculturation process seems to indicate that international students may go 
through acculturative complications which may not be easily managed through cultural conformity.

\section{Depression}

According to American Psychiatric Association (cited in Arslan, Ayranci, Unsal, \& Arslantas, 2009), depression is a frequent and severe mental health problem characterized by loss of interest, depressed mood, low self-worth, sleep difficulties, poor concentration, feelings of guilt and low energy which unsympathetically affect person's thoughts, feelings and actions. Depressive symptoms can be chronic and persistent and lead to substantial impairments in an individual's ability to undertake his or her day to day responsibilities. As depression is a widespread problem that can affect a wide range of groups such as males, females, students, workers, adolescents (Pilgrim, Rogers, \& Pescosolido, 2011), several research findings indicate that depression is a common psychological problem among students population of institutions for higher education (e.g., Beiter et al., 2015; Shamsuddin et al., 2013). In a systematic review of studies on depression prevalence among university students, the rate of prevalence among university students was also found substantially higher than those found in general population (Ibrahim, Kelly, Adams, \& Glazebrook, 2013).

Research works on depression among international students are limited (Sümer, Poyrazli, \& Grahame, 2008). However, some studies revealed that international students are prone to health problems like anxiety and depression because of a myriad of adjustment difficulties. For example, in a study conducted on depressive symptoms among international university students in northern Japan, the students were found experiencing depressive symptoms (Eskanadrieh et al., 2012). Similarly, in a survey study conducted in the Philippines, higher levels of depressive symptoms were detected among international students due to social and demographic factors such as level of satisfaction with one's financial condition, and level of closeness with parents and peers (Romeo, Madelene, Susana, \& Cristina, 2013).

\section{Cultural Intelligence}

Ang and Van Dyne (2008) defined cultural intelligence as an individual's capability to function commendably in a multi-cultural context. According to Livermore (2011), cultural intelligence refers to an individual's ability to effectively function in contexts characterized by different national, ethnic and organizational cultural practices. More to these 
definitions, cultural intelligence refers to the person's ability to interact effectively with people of different cultures (Crowne, 2008). The commonality between the definitions is that cultural intelligence is a unique individual quality that allows people to better function in cross-cultural settings. In the current study, it refers to the ability of international students to adequately adjust to the new social, cultural and educational environment of the host community. Cultural intelligence is believed to have an ameliorating impact on peoples' psychological adjustment to the new environment.

According to Earley and Ang (2003), cultural intelligence is a multidimensional construct that comprises of four facets: metacognitive, cognitive, motivational and behavioral. The metacognitive branch of cultural intelligence refers to how an individual makes sense of the multicultural experiences. The cognitive aspect of cultural intelligence refers to the general knowledge structures and cognitive maps about different cultural practices such as legal and economic systems, sociocultural values and norms. The motivational cultural intelligence refers to the individual's passion, interest, appreciation, and enthusiasm in acquiring and experiencing multiple cultural practices through having a positive interaction with people of different cultural upbringings. The behavioral aspect of cultural intelligence refers to the individual's competency to navigate both verbal and non-verbal actions that appropriately fit the multicultural interactions.

\section{Prior Travel Experience, Age, Acculturative Stress, and Depression}

Prior travel experience has long been thought to contribute to psychological adjustment positively because previous abroad exposure enables individuals to have a clear expectation about the potential adjustment difficulties that they could encounter in the host environment (Black, Mendenhall, \& Oddou, 1991). It seems that individuals with previous international experience have the advantage of making the best use of their experience in better functioning in a culturally diverse environment. Despite the paucity of empirical studies conducted among international students, only a few of them are in favor of the protective role of students' previous travel experience against their adverse psychological acculturative outcomes (Akhtar \& Kröner-Herwig, 2015).

Age is also another personal characteristic that affects students' acculturative experiences. The nature of the relationship between age and acculturative stress and depression is unclear and controversial. On one hand, some researchers argue that young individuals are not mature and 
competent enough to effectively deal with acculturative encounters and consequently become more prone to acculturative stress and depression. On the other hand, according to Berry (1997), young students are believed to better function and quickly adjust to new environment because of their flexibility and openness to learning the new lifestyles (e.g., language).

\section{Cultural Intelligence, Acculturative Stress, and Depression}

Studies on cultural intelligence make an effort to clarify the reason why some individuals adapt more effectively to new intercultural setting than others. Cultural intelligence is one of the most prominent factors that profoundly influences the quality of the adjustment process to the new environment, that is to say individuals with high cultural intelligence tend to make a successful adjustment ( $\mathrm{Ng} \&$ Earley, 2006) with minimal experience of acculturative stress (Richard, Reginald, \& Brent, 2006). Similarly, Le, Jiang, and Nielsen (2016) argue that cultural intelligence guides people in adjusting to the multicultural setting and handling stress, cultural barriers and difficulties in cross-cultural communications and interactions. Despite the limited empirical evidence for the relationship between cultural intelligence and acculturative stress, some studies have demonstrated that cultural intelligence is negatively related to acculturative stress. For instance, Khan (2015) conducted a study on 200 acculturating students in India and provided empirical evidence for the predicting impact of cultural intelligence on acculturative stress.

Tamannaeifar and Hesampour (2016) also found cultural intelligence to significantly contribute to students' adaptability to the university environment. Cultural intelligence guides the entire student population within and outside one's native country migrant student population to effectively function in acculturative processes. However, it has paramount importance on international students' psychological and sociocultural adjustments. In line with this argument, cross-cultural studies have also asserted that cultural intelligence significantly predicts the feeling of acculturative stress of the international students in the multicultural environment (Ayoob, Wani, Ahmad, Jan, \& Dar, 2015). Such empirical studies indicate that cultural intelligence facilitates the process of acculturation towards the new environment and thereby shelters students from experiencing a plethora of harmful acculturative outcomes like stress and tension.

Previous cultural intelligence studies have informed us that cultural intelligence is positively associated with psychological well-being and 
negatively associated with mental health problems such as depression. For example, Elyasi et al. (2017) carried out a cross-sectional study of 385 university students to examine the relationship between cultural intelligence and mental health. Their report reveals that there is a significant relationship between cultural intelligence and mental health. In a similar recent study, cultural intelligence was also found to moderate the relationship between acculturative stress and somatic symptoms of general health, meaning that, the relationship between acculturative stress and somatic symptoms was stronger when cultural intelligence was low, but the relationship was weak when the level of cultural intelligence was high (Ayoob \& Alsultan, 2017). In line with these findings, cultural intelligence moderated the relationship between culture shock, one component of acculturative stress, and psychological and sociocultural adaptation (Presbitero, 2016).

Additionally, a study on the relationship between cultural intelligence and psychological well-being with the moderating impact of mindfulness among 110 international students in Taiwan was carried out and reported. The report revealed that the metacognitive and behavioral components of cultural intelligence were positively and significantly correlated with psychological well-being (Yang \& Chang, 2017). However, overall cultural intelligence was not significantly correlated with psychological well-being. Harrison and Brower (2011) conducted a study on the role of cultural intelligence and personality hardiness on homesickness by surveying 537 international students in the United States of America. Their results revealed that personality hardiness and all dimensions of cultural intelligence significantly predicted psychological adjustment; in particular, the emotional aspect of cultural intelligence depicted the most potent power of predicting homesickness. This result signposts the vital role of cultural intelligence in psychological adjustments, although the study examined only one aspect of acculturative stress (i.e., homesickness) as a measure of acculturative difficulties and which may not give a complete picture of adjustment difficulties.

Therefore, from the previous literature, it can generally be inferred that cultural intelligence, age, and prior travel experience impact acculturative stress and depression of the international students even though the studies are insufficient and some have controversial and mixed-up results. 


\section{Acculturative Stress as a Mediator Between Cultural Intelligence and Depression}

Some studies have analyzed the mediating effect of acculturative stress on the relationship between predictors and mental health outcomes. For instance, acculturative stress was found to function as a mediator on the relationship between the acculturation and mental distress and depression, indicating that a low level of acculturation intensified the level of acculturative stress and thereby led to debilitating mental health problems such as depressive symptoms (Cho, Jang, Ko, Lee, \& Moon, 2017). Similarly, acculturative stress was found to mediate the connection between cultural orientation and psychological functioning, suggesting that balanced cultural orientation towards both the native and host culture shields individual students from higher acculturative stress and subsequently buffers against exacerbating mental health outcomes and can enhance positive health outcomes (Du, Li, Lin, \& Tam, 2015).

\section{Statement of the Problem}

Acculturative stress, anxiety, and depression are appearing to be prevalent health problems among the international students as a result of adjustment difficulties in a new environment. Given this, there is a need to have an accurate and comprehensive understanding of the antecedents of psychological adjustment and thereby to find out strategies that have the potential of safeguarding students from detrimental acculturative outcomes. For this purpose, researchers have uncovered various psychological resources such as coping skills, social support, self-efficacy, emotional and cultural intelligence, and personality which act as vital influencing factors of psychological adaptation (Mesidor \& Sly, 2016). However, empirical studies about cultural intelligence on international students have globally been unheeded and lagged behind (Wang, Heppner, Wang, \& Zhu, 2015).

Likewise, the international students' adjustment studies from individuallevel perspective are also limited in China although the number of students in the country is swiftly increasing (Wen, Hu, \& Hao, 2017). Drawing on the prevailing mental health problems among the students and the limited cultural intelligence studies, the present study primarily endeavored to address the gap by examining the relationships between cultural intelligence, acculturative stress and depression. Understanding the relationship between the study variables may provide practical implication for university communities in assisting international students to better adjust 
to the new environment and thereby fostering their psychological health and well-being.

\section{Research Hypotheses}

Following the reviewed literature and the theoretical conceptualization of cultural intelligence and acculturative stress, the study voiced the following hypotheses.

Hypothesis 1: Students with prior international travel experience before coming to China have lower acculturative stress and depression than students with no previous travel exposure.

Hypothesis 2: Younger students have lower acculturative stress and depression than older students.

Hypothesis 3: Students' cultural intelligence negatively relates to acculturative stress.

Hypothesis 4: Students' cultural intelligence negatively relates to depression.

Hypothesis 5: Acculturative stress plays mediating role to the relationship between cultural intelligence and depression.

\section{METHOD}

\section{Participants}

The study recruited a total of 506 volunteer international university students (44\% females) with the average age of 27.32. Participants were conveniently selected from seven universities located in Wuhan, a city located in central China. Their average length of stay in the host country was 21 months. $61 \%$ of them had prior international travel experiences. $70 \%$ of the participants were single while the rest of $30 \%$ were married. The frequency distribution of the respondents as per educational status was $30 \%$ Bachelors, $41 \%$ Masters and 29\% Doctorate. Majority of the participants were from Asia (45\%), and Africa (41\%), and $14 \%$ were from the rest of the continents (Europe, Oceania, Latin America, and North America). To determine the sample size, the minimum required sample size using Yamane (1973)'s formula with the margin of error of 5\% was calculated. Out of the total population of around 14, 000 international students studying in the seven targeted universities in 2018, the calculated 
sample size is 388.9 . The sample size of the current study was 506 , which is higher than the calculated sample size.

\section{Measures}

\section{Cultural Intelligence}

The study employed Ang et al. (2007)'s Cultural Intelligence Scale (CQS) to measure cultural intelligence. CQS is a reliable 20 item multifaceted scale encompassing four subscales rated on seven points Likert scales ranging from 1 (Strongly Disagree) to 7 (Strongly Agree). The four subscales of CQS are Metacognition ( $4-$ items, $\alpha=.72)$, Cognition ( $6-$ items, $\alpha=.86$ ), Motivation ( 5 - items, $\alpha=.76$ ), and Behavior ( $5-$ items, $\alpha=$ .83). Higher scores reflect a higher level of cultural intelligence. Several studies supported the reliability of the instrument (Khan, 2015; Yang \& Chang, 2017). Some studies provided evidence for the predictive, discriminant and convergent validity of the measure (e.g., Ang et al., 2007; Khan \& Hasan, 2016).

\section{Acculturative Stress}

The study adopted Acculturative Stress for International Students Scale (Sandhu \& Asrabadi, 1994) to measure the acculturative stress. The device is a 36- items self-report questionnaire rated on 5 points Likert scale ranging from 1 (Strongly Disagree) to 5 (Strongly Agree). Higher scores represent the prevalence of higher level of acculturative stress. Several researchers have agreed that the measure is a highly reliable instrument with reliability coefficient, ranging from .92 to .94 (e.g., Liu et al., 2016; Sandhu \& Asrabadi, 1994; Wei et al., 2007). Besides, Sandhu and Asrabadi (1994) provided validity evidence for the instrument.

\section{Depression}

Radloff (1977)' Center for Epidemiological Studies Depression Scale (CES-D) was used to measure depression. CES-D is a self-report instrument devised to measure depressive symptoms such as depressed mood, the feeling of worthlessness, loss of appetite, poor concentration and sleep disturbance in the general population. CES-D contains 20 both positively and negatively worded items rated on four points Likert scale which ranges between 1 (Rarely or none of the time) and 4 (All of the time). Higher scores 
reflect a prevalence of higher depressive symptoms. CES-D has consistently been reported to have high Cronbach's alpha coefficients ranging from .85 to .90 across studies (Radloff, 1977).

\section{Data Collection Procedure}

Once the study was approved by the School of Psychology, Central China Normal University, anonymous self-report questionnaires were distributed to the participant students in their respective schools and classrooms. Some senior Master or Ph.D. students from Central China Normal University involved in administering, guiding and facilitating the data gathering process in case participants may face difficulties in understanding the item statements. According to the ethical rules of psychological research in China, participation in the present study was voluntary and based upon informed consent. Participants were also assured that the data would not be used for any other purpose than the research.

\section{RESULTS}

\section{Descriptive Statistics of the Study Variables}

Table 1 presented the summary of the means, standard deviations and reliability coefficients of the valuable measures in the study. Reliability of the instruments was established by Cronbach's alpha. The study measures were found to have sufficient internal consistency, reflecting that the data obtained from this scale was trustworthy. Besides, to assess the assumption of normality for the study variables, measures of distribution shape, skewness and kurtosis were calculated. The values for skewness and kurtosis between -2 and +2 are considered as acceptable limits to prove the normal univariate distribution (Gravetter \& Wallnau, 2014). Therefore, as depicted in Table 1, the values of skewness and kurtosis for all study variables fell within the acceptable range, reflecting that data were normality distributed. 
Table 1: Summary of Descriptive Statistics of the Study Variables

\begin{tabular}{llllll}
\hline Variables & alpha & $M$ & $S D$ & $S k$ & $K u$ \\
\hline $\begin{array}{l}\text { Cultural } \\
\text { intelligence }\end{array}$ & 0.88 & 4.65 & 0.86 & 0.00 & -0.09 \\
$\begin{array}{l}\text { Acculturative } \\
\text { stress }\end{array}$ & 0.92 & 2.27 & 0.57 & -0.08 & -0.49 \\
\begin{tabular}{l} 
Depression \\
\hline
\end{tabular} & 0.82 & 1.88 & 0.41 & 0.41 & -0.32 \\
\hline
\end{tabular}

The summary of the inter-correlations between the study variables are presented in Table 2. The results indicated that students with no previous travel experience as well as older students reported greater acculturative stress, and students with no previous travel experiences as well as younger students reported higher depression. Cultural intelligence was found to be negatively related to both acculturative stress $(r=-0.27, p<.01)$ and depression $(r=-0.18, p<.01)$, delineating that students with a higher level of cultural intelligence were found to have lower acculturative stress and fewer depressive symptoms.

\section{Bivariate Correlations Between Study Variables}

The summary of the inter-correlations between the study variables are presented in Table 2. The results indicated that students with no previous travel experience as well as older students reported greater acculturative stress, and students with no previous travel experiences as well as younger students reported higher depression. Cultural intelligence was found to be negatively related to both acculturative stress $(r=-0.27, p<.01)$ and depression $(r=-0.18, p<.01)$, delineating that students with a higher level of cultural intelligence were found to have lower acculturative stress and fewer depressive symptoms.

\section{Table 2: Summary of Inter-correlations Between Study Variables}

\begin{tabular}{lllll}
\hline Variables & 1 & 2 & 3 & 4 \\
\hline Age & 1.00 & & & \\
Prior Travel Experience & 0.03 & 1.00 & & \\
Cultural Intelligence & 0.01 & $0.18^{* *}$ & 1.00 & \\
Acculturative stress & $0.16^{* *}$ & $-0.19^{* *}$ & $-0.27^{* *}$ & 1.00 \\
Depression & $-0.10^{*}$ & $-0.10^{*}$ & $-0.18^{* *}$ & $0.37^{* *}$ \\
\hline Note. ${ }^{*} p<0.05,{ }^{* *} p<0.01$. For Prior travel experience: Yes $=1$, No $=0$
\end{tabular}




\section{Predicting Acculturative Stress from Age, Prior Travel Experience and Cultural Intelligence}

The hierarchical multiple regression analysis was run to examine the predicting effect of age, prior travel experience, and cultural intelligence. Predicting variables were sequentially entered into the equations. Age and prior abroad travel experience were put into the first block. With the variables of the first bock already inputted, cultural intelligence was entered into the second bock. The results were presented in table 3.The results indicated that the combined effect for block one variables accounted for $7 \%$ of the variance in acculturative stress. Students with prior travel experience $(\beta=-0.20, p<001)$ and younger students $(\beta=0.17, p<001)$ reported less acculturative stress. When cultural intelligence was added into the second block, the overall model explained $12 \%$ variance. After controlling the effects of age and prior travel experience, cultural intelligence explained additional $5 \%$ of the variance in acculturative stress and this additional contribution was statistically significant. Students with higher cultural intelligence experienced lower level of acculturative stress $(\beta=-0.24, p<$ $.001)$. Prior travel experience $(\beta=-0.16, \mathrm{p}<.01)$ and age $(\beta=0.17, \mathrm{p}<.01)$ continued to maintain their significant unique contribution in the second bock.

\section{Predicting Depression from Age, Prior Travel Experience, Cultural Intelligence and Acculturative Stress}

Similar with the above analysis, participants' age and prior travel experience were put into the first block of the model followed by adding acculturative into the second block and cultural intelligence into the third block. As depicted in Table 3, findings of the analyses noticed that students' age and prior travel experience tallied to explain $2 \%$ of the variance in depression. Students who had the experience of travelling abroad $(\beta=-0.10$, $p<.05)$ and older students $(\beta=-0.11, p<.05)$ appeared to have fewer depressive symptoms. Including cultural intelligence into the second block of the model improved the predicting power of the overall model to account for $4 \%$ of the variance in the outcome variable and this was statistically significant. Cultural intelligence additionally contributed $2 \%$ of variance in depression statistically significantly. Culturally intelligent students reported less depressive symptoms $(\beta=-.16, p<.001)$. Compared to block one, age continued to maintain its significant unique contribution to the outcome variable in the second model. 
Table 3: Summary of Hierarchical Regression for Predictors of Acculturative Stress and Depression

\begin{tabular}{|c|c|c|c|c|c|}
\hline \multirow[b]{2}{*}{ Predictors } & \multicolumn{2}{|c|}{$\begin{array}{c}\text { Outcome: } \\
\text { Acculturative stress }\end{array}$} & \multicolumn{3}{|c|}{$\begin{array}{l}\text { Outcome: } \\
\text { Depression }\end{array}$} \\
\hline & Equation1 & Equation2 & Equation 1 & Equation2 & Equation3 \\
\hline Age & $0.17^{* * *}$ & $0.17^{* * * *}$ & $-0.11^{*}$ & $-0.11^{*}$ & $-0.17^{* * * *}$ \\
\hline $\begin{array}{l}\text { Prior Travel } \\
\text { Experience }\end{array}$ & $-0.20^{* * *}$ & $-0.16^{* * *}$ & $-0.10^{*}$ & -0.07 & -0.01 \\
\hline $\begin{array}{l}\text { Cultural } \\
\text { intelligence }\end{array}$ & & $-0.24^{* * *}$ & & $-0.16^{* * *}$ & -0.07 \\
\hline $\begin{array}{l}\text { Acculturative } \\
\text { stress }\end{array}$ & & & & & $0.38^{* * * *}$ \\
\hline$R^{2}$ change & 0.07 & 0.05 & 0.02 & 0.02 & 0.13 \\
\hline F change & $17.60^{* * *}$ & $22.63^{* * *}$ & $5.52^{*}$ & $8.03^{* * * *}$ & $26.30^{* * *}$ \\
\hline
\end{tabular}

Note. For Prior travel experience: Yes $=1$, No $=0 . * p<0.05 ; * * p<0.01$; $* * * p<0.001$. Regression coefficients were standardized.

When the acculturative stress was added into the third bock, the overall model explained $18 \%$ of the variance in depression and the regression equation was statistically significant. After controlling the effects of age, prior travel experience, and cultural intelligence, acculturative stress contributed an additional $13 \%$ of the variance in depression. The additional contribution was statistically significant as well. This result indicated that international students with high level of acculturative stress experienced greater depressive symptoms than the ones with lower acculturative stress. The unique effect of age on depression in block three was still significant, while the unique effect of prior travel experience on depression was reduced to be insignificant, indicating that the effect of prior travel experience was mediated by cultural intelligence and acculturative stress.

\section{Mediating Effect of Acculturative Stress on the Relationship Between Cultural Intelligence and Depression}

The mediating effect was tested with the PROCESS macro for SPSS (Hayes , 2013) with controlling age and prior travel experience. As displayed in figure 1, cultural intelligence predicted both the mediating variable of acculturative stress $(a=-.16, t=-5.68, p<.001)$ and outcome 
variable of variable of depression $(c=-.08, t=-3.69, p<.001)$. The mediator also predicted the dependent variable $(b=.28, t=8.76, p<.001)$. The direct effect of cultural intelligence on depression was no longer $\operatorname{significant}\left(c^{\prime}=-.03, t=-1.69, p=.09\right)$. The indirect effect was $(-0.16)(0.28)$ $=-0.04$. To assess the significance of the indirect effect, bias-corrected $95 \%$ CI was computed based on 5000 bootstrapping resample, and the indirect effect was $-0.04, \mathrm{SE}=.01,95 \% \mathrm{CI}$ was from -0.07 to -0.03 . This interval does not overlap with zero denotes significant mediation.

\section{Figure 1: Mediation Path Diagram}

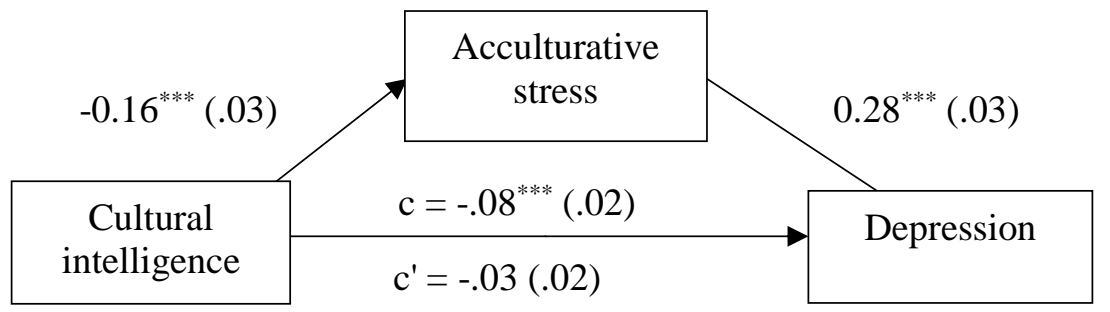

Note. Unstandardized coefficients, with standard errors in parentheses. $* * * p<.001$

\section{DISCUSSION}

The study examined how age, prior travel experience and cultural intelligence impact acculturative psychological outcomes (i.e., acculturative stress and depression). In line with the hypothesis that age and prior travel experience predict acculturative stress and depression, the present finding confirmed that students with previous exposure experienced lower acculturative stress and depression than their counterparts. Several studies supported the present finding that previous abroad exposure decreased students' feeling of stress (Akhtar \& Kröner-Herwig, 2015; Smiljanic, 2017) and depression. It is not a surprise that students with previous travel experience reported lower acculturative stress and depression because those students have already experienced how it felt to be very far from home. They might have also gained the skill and competence on how to adjust to the new environment because of their clear expectation and visualization about the host environment (Smiljanic, 2017). Pertaining to age, the study found younger students to experience less acculturative stress which is in line with Berry's (1997) argument that young individuals easily integrate with mainstream society. Despite their better adjustment to the new 
averment, younger students reported greater depressive symptoms than older students. The reason for this might be attributed to the psychological and emotional immaturity of younger students to successfully cope with emotional disturbances, frustration, and hopelessness which might be stemmed from factors that are not associated with adjustment difficulties such as academic failures, and relationship problems. Even though there is a scarcity of depression studies among international students (Sümer et al., 2008), some studies reported that the rate of prevalence of depression is relatively common among the students irrespective of their age (Eskanadrieh et al., 2012).

Following the acculturative stress models of Berry (2003) and cultural intelligence model of Ang and Van Dyne (2008), the study conjectures that cultural intelligence predicts acculturative stress and depression assuming that students who are culturally aware before they come into the first contact with the new society are likely to experience less acculturative difficulties and depression. The finding of the study corroborated this hypothesis that international students with a higher level of cultural intelligence before they sojourn to the host community reported less acculturative stress and depression. A possible explanation for this finding goes to the theoretical postulation of cultural intelligence as part of general intelligence and acculturative stress. Berry (2003), in his acculturation theory, argued that psychological resources that individuals bring to the arena of acculturation are essential features of psychological acculturation.

In their cultural intelligence model, Ang and Van Dyne (2008) conceptualized cultural intelligence as a mental ability that enables individuals to control and be aware of their cognitive processes in dealing with people of different cultures. It also enables them to critically and consciously examine their own culture in order to make a possible amendment to better fit in with the host culture (Van Dyne et al., 2012). According to Ang and Van Dyne (2008), the ability of individuals to gain comprehensive knowledge and understanding of the host community's cultural practices allows them to develop a sense of cultural recognition and appreciation which notably orchestrates and guides their social interaction within the culture. Subsequently, individuals who have higher cognitive cultural intelligence are believed to better function and develop healthy relationships with culturally diverse people. Motivational cultural intelligence enables international students to obtain the necessary willingness to acquire important cultural information and learn from other culturally diverse students (Van Dyne et al., 2012). Besides, motivational cultural intelligence encapsulates individuals' internal motivation as a 
source of personal satisfaction, external motivation as a source of concrete benefits, and self-efficacy as an opportunity to demonstrate their potential and capabilities by taking part in different intercultural experiences (Van Dyne et al., 2012). Behavioral cultural intelligence also helps individuals to depict appropriate verbal and nonverbal behaviors during sociocultural interactions with people of different cultural backgrounds (Ang \& Van Dyne, 2008). Behavioral cultural intelligence is the most salient dimension of cultural intelligence because it enables individuals to gain a sense of control and regulation over their social behaviors in new multicultural setting with minimal misunderstanding and attributionl problems (Ghahremani, Monterosso, Jentsch, Bilder, \& Poldrack, 2010).

Several empirical studies have also supported the current finding that cultural intelligence is inversely related to adverse acculturative outcomes (Harrison \& Brower, 2011; Khan, 2015; Lin, Chen, \& Song, 2012). TzuPing and Wei-Wen (2017) also found metacognitive and behavioral aspects of cultural intelligence to be significant predictors of psychological wellbeing. However, unlike the present finding, some international students' studies found no significant explained variance for the cultural intelligence on acculturative psychological outcomes (Yang \& Chang, 2017; Ward, Fischer, Zaid Lam, \& Hall, 2008) which might due to the self-report nature of the cultural intelligence (Ward et al., 2008).

The sample data also confirmed the hypothesis that acculturative stress mediated the relationship between cultural intelligence and depression, indicating that students with high level of cultural intelligence experienced lower level of acculturative stress and subsequently fewer depressive symptoms. Despite the limited available literature, parallel to the present finding, some related empirical studies showed that acculturative stress mediated the relationship between psychological adjustment outcome and its predictors such as collectivistic cultural orientation (Du et al., 2015), and acculturation (Cho et al., 2017).

Despite its contribution, the study had several limitations. First, as the study was descriptive cross-sectional and examined all the study variables at the same point in time, causal relationships between the variables were not established. Therefore, future studies might continue to study the variables using other research designs such as longitudinal or quasi-experimental approaches. Secondly, the participants recruited in the study were international students completing their studies in universities located in Wuhan which might affect the external validity of the findings. Since participants of the study were also from a wide range of countries, their diversified cultural background might affect the finding. Therefore, future 
research could endeavor to focus on a specific group of international students with sufficient sample representativeness to achieve a comprehensive and accurate understanding of the psychological adjustment of the students. Finally, participants' response bias might affect the accuracy of the findings, and future studies might, therefore, replicate the study using other research design which might minimize the potential response bias of participants.

\section{IMPLICATIONS}

There are no considerable empirical cultural intelligence studies on international students. Therefore, the findings of the present study could be noteworthy in theoretically enriching the scientific body of cross-cultural knowledge by examining 1) the predicting effects of cultural intelligence, age and prior travel experience on acculturative stress and depression; 2) the mediating role of acculturative stress on the relationship between cultural intelligence and depression.

Practically, the significantly explained variance of the cultural intelligence in both acculturative stress and depression could guide university communities to better deal with adjustment difficulties of their international students. The findings of the study inform university personnel interacting with international students to create suitable ground where students with low level of cultural intelligence cultivate and capitalize their ability to effectively function in a multicultural context and thereby promote their mental health. For instance, university communities can arrange various curricular and co-curricular activities which include educational clubs and units (e.g. language club, research and publication units), alumni programs, cultural exchange festivals, and sport activities, which can all take place inside the campus and outside the campus with other partner universities. Besides, the universities can have their campus magazines which actively work on publishing students' multicultural experiences and reflections. Such activities also seem to help students feel confident and capable of mobilizing their full attention and energy towards active engagement and learning in the process of acculturation while respecting, appreciating and valuing cultural diversity. 


\section{CONCLUSION}

The present study provided statistical evidence that the cultural intelligence, age and prior travel experience have significant predicting effects on both acculturative stress and depression. The findings of the study underline that cultural intelligence, age, and prior travel experience are salient influence factors of psychological adjustment. The mediating effect of acculturative stress on the cultural intelligence-depression relationship also highlights the importance of considering potential mediating factors in scientific understanding of the acculturative context of international students.

\section{REFERENCES}

Akhtar, M., \& Kröner-Herwig, B. (2015). Acculturative stress among international students in context of socio-demographic variables and coping styles. Current Psychology, 34(4), 803-815.

Ang, S., \& Van Dyne, L. (2008). Handbook of cultural intelligence: Theory, measures and applications. Armonk: M.E. Sharpe.

Ang, S., Van Dyne, L., Koh, C., Ng, K. Y., Templer, K. J., Tay, C., \& Chandrasekar, N. A. (2007). Cultural intelligence: Its measurement and effects on cultural judgment and decision making, cultural adaptation and task performance. Management and Organization Review, 3(3), 335-371.

Arslan, G., Ayranci, U., Unsal, A., \& Arslantas, D. (2009). Prevalence of depression, its correlates among students, and its effect on healthrelated quality of life in a Turkish university. Upsala Journal of Medical Sciences, 114(3), 170-177.

Ayoob, M., \& Alsultan, A. (2017). Cultural intelligence as a moderator of acculturative stress to health among collegestudents. International Journal of Science and Research, 6(4), 1905-1910.

Ayoob, M., Wani, N., Ahmad, M., Jan, M., \& Dar, B. (2015). Cultural intelligence as a predictor of acculturative stress and psychological well-being among college students. Journal of the Indian Academy of Applied Psychology, 41(1), 86-94.

Beiter, R., Nash, R., McCrady, M., Rhoades, D., Linscomb, M., Clarahan, M., \& Sammut, S. (2015). The prevalence and correlates of depression, anxiety, and stress in a sample of college students. Journal of Affective Disorders, 173(Supplement C), 90-96.

Berry, J. W. (1997). Immigration, acculturation, and adaptation. Applied Psychology: An International Review, 46(1), 5-34. 
Berry, J. W. (2003). Conceptual approaches to acculturation. In K. M. Chun, P. Balls Organista, \& G. Marín (Eds.), Acculturation: Advances in theory, measurement, and applied research (pp. 17-37). Washington, DC: American Psychological Association.

Berry, J. W. (2005). Acculturation: Living successfully in two cultures. International Journal of Intercultural Relations, 29(6), 697-712.

Bevis, T. B. (2002). At a glance: International students in the United States. International Educator, 11(3), 12-17.

Bista, K. (2019). Exploring the field: Understanding the international student experience. In K. Bista (Ed.), Global perspectives on international student experiences in higher (pp. 1-16). New York, NY: Routledge.

Black, S. J., Mendenhall, E. M., \& Oddou, G. (1991). Toward a comprehensive model of international adjustment: An integration of multiple theoretical perspective. The Academy of Management Review, 16(2), 291-317.

Brunsting, N. C., Zachry, C., \& Takeuchi, R. (2018). Predictors of undergraduate international student psychosocial adjustment to US universities: A systematic review from 2009-2018. International Journal of Intercultural Relations, 66, 22-33.

Cho, Y. J., Jang, Y., Ko, J. E., Lee, S. H., \& Moon, S. K. (2017). Acculturation, acculturative stress, and depressive symptoms in international migrants: A Study with vietnamese women in South Korea. Journal of Immigrant and Minority Health, 20(5), 11031108.

Crowne, K. A. (2008). What leads to cultural intelligence? Business Horizons, 51(5), 391-399.

Du, H., Li, X., Lin, D., \& Tam, C. C. (2015). Collectivistic orientation, acculturative stress, cultural self-efficacy, and depression: A longitudinal study among Chinese internal migrants. Community Mental Health Journal, 51(2), 239-248.

Earley, P. C., \& Ang, S. (2003). Cultural intelligence: Individual interactions across cultures. Palo Alto, CA: Stanford University Press.

Elyasi, F., Ahmadi, M., Mohsenimoalemkoale, N., Jafari, S. N., Hoseini, S. M., \& Hoseini, S. H. (2017). Relationship between cultural intelligence and mental health in students of the Medical University of Mazandaran in 2017. Future of Medical Education Journal, 7(3), 34-39. 
Eskanadrieh, S., Liu, Y., Yamashina, H., Kono, K., Arai, A., B. Lee, R., \& Tamashiro, H. (2012). Depressive symptoms among international university students in northern Japan: Prevalence and associated factors. Journal of International Health, 27(2), 165-170.

Ghahremani, D. G., Monterosso, J., Jentsch, J. D., Bilder, R. M., \& Poldrack, R. A. (2010). Neural components underlying behavioral flexibility in human reversal learning. Cerebral Cortex, 20(8), 1843-1852.

Gravetter, F., \& Wallnau, L. (2014). Essentials of Statistics for the Behavioral Sciences $\left(8^{\text {th }}\right.$ ed.). Belmont, CA: Wadsworth.

Harrison, J. K., \& Brower, H. H. (2011). The impact of cultural intelligence and psychological hardiness on homesickness among study abroad students. The Interdisciplinary Journal of Study Abroad, 21, 31-62.

Hayes, A. F. (2013). Introduction to mediation, moderation, and conditional process analysis: A regression-based approach. New York, NY:Guilford Press Publications.

Ibrahim, A. K., Kelly, S. J., Adams, C. E., \& Glazebrook, C. (2013). A systematic review of studies of depression prevalence in university students. Journal of Psychiatric Research, 47(3), 391-400.

Khan, K. (2015). Predicting effect of cultural intelligence on acculturative stress. Journal of Ravishankar University, Part-A, 23-29.

Khan, K., \& Hasan, B. (2016). Validation of the 20-item cultural intelligence scale in Indian within country migrated students. The International Journal of Indian Psychology, 3(2), 14-23.

Krämer, A., Pröfer-Krämer, L., Stock, C., \& Tshiananga, J. T. (2004). Differences in Health Determinants Between International and Domestic Students at a German University. Journal of American College Health, 53(3), 127-132.

Le, H., Jiang, Z., \& Nielsen, I. (2018). Cognitive cultural intelligence and life satisfaction of migrant workers: The roles of career engagement and social injustice. Social Indicators Research, 139(1), 237-257.

Lin, Y., Chen, A. S., \& Song, Y. (2012). Does your intelligence help to survive in a foreign jungle? The effects of cultural intelligence and emotional intelligence on cross-cultural adjustment. International Journal of Intercultural Relations, 36(4), 541-552.

Liu, Y., Chen, X., Li, S., Yu, B., Wang, Y., \& Yan, H. (2016). Path analysis of acculturative stress components and their relationship with depression among international students in China. Stress and Health, 32(5), 524-532. 
Livermore, D. (2011). The cultural intelligence difference: Master the one skill you can't do without in today's globabl economy. New York, NY: AMACOM.

Mesidor, J. K., \& Sly, K. F. (2016). Factors that contribute to the adjustment of international students. Journal of International Students, 6(1), 262-282.

Mori, S. C. (2000). Addressing the mental health concerns of international students. Journal of Counseling and Development, 78(2), 137-143.

Mustaffa, C. S., \& Ilias, M. (2013). Relationship between students adjustment factors and cross cultural adjustment: A survey at the northern university of Malaysia. Intercultural Communication Studies, 1(22), 279-300.

Ng, K.-Y., \& Earley, P. C. (2006). Culture and intelligence: Old constructs, new frontiers. Group \& Organization Management, 31(1), 4-19.

Pilgrim, D., Rogers, A., \& Pescosolido, B. (2011). The SAGE handbook of mental health and illness. London, Sage.

Presbitero, A. (2016). Culture shock and reverse culture shock: The moderating role of cultural intelligence in international students' adaptation. International Journal of Intercultural Relations, 53(Supplement C), 28-38.

Radloff, L. S. (1977). The CES-D Scale: A self-report depression scale for research in the general population. Applied Psychological Measurement, 1(3), 385-401.

Richard, B., Reginald, W., \& Brent, M. (2006). Cultural intelligence: Understanding behaviors that serve people's goals. Group \& Organization Management, 31(1), 40-55.

Romeo, L., Madelene, M., Susana, E., \& Cristina, R. (2013). Factors associated with higher levels of depressive symptoms among international university students in the Philippines. Southeast Asian journal of tropical medicine and public health, 44(6), 10981107.

Sandhu, D. S., \& Asrabadi, B. R. (1994). Development of an acculturative stress scale for international students: preliminary findings. Psychological Reports, 75(1), 435-448.

Shamsuddin, K., Fadzil, F., Wan Ismail, W. S., Shah, S. A., Omar, K., Muhammad, N. A., . . Mahadevan, R. (2013). Correlates of depression, anxiety and stress among Malaysian university students. Asian Journal of Psychiatry, 6(4), 318-323.

Smiljanic, I. (2017). The role of attachment, travel experiences and english proficiency in international students' acculturative stress and 
depressive symptoms Journal of International Students, 7(2), 188203.

Sümer, S., Poyrazli, S., \& Grahame, K. (2008). Predictors of depression and anxiety among international students. Journal of Counseling \& Development, 86(4), 429-437.

Tamannaeifar, M., \& Hesampour, F. (2016). The relationship between cultural and emotional intelligence with students' adjustment to university. International Academic Journal of Organizational Behavior and Human Resource Management and Organization Review, 3(3), 1-13.

Yang, T., \& Chang, W. (2017). The relationship between cultural intelligence and psychological well-being with the moderating effects of mindfulness: A study of international students in Taiwan. European Journal of Multidisciplinary Studies, 5(1), 384-391.

Van Dyne, L., Ang, S., Yee Ng, K., Rockstuhl, T., Ling Tan, M., \& Koh, C. (2012). Sub-dimensions of the four factor model of cultural intelligence: Expanding the conceptualization and measurement of cultural intelligence. Social and Personality Psychology Compass, 6(4), 295-313.

Wang, K. T., Heppner, P. P., Wang, L., \& Zhu, F. (2015). Cultural intelligence trajectories in new international students: Implications for the development of cross-cultural competence. International Perspectives in Psychology: Research, Practice, Consultation, 4(1), 51-65.

Ward, C., Bochner, S., \& Furnham, A. (2005). The psychology of culture shock. London: Routledge.

Ward, C., Fischer, R., Zaid Lam, F. S., \& Hall, L. (2008). The convergent, discriminant, and incremental validity of scores on a self-report measure of cultural intelligence. Educational and Psychological Measurement, 69(1), 85-105.

Wei, M., Heppner, P. P., Mallen, M. J., Ku, T.-Y., Liao, K. Y.-H., \& Wu, T.-F. (2007). Acculturative stress, perfectionism, years in the united states, and depression among Chinese international students. Journal of Counseling Psychology, 54(4), 385-394.

Wen, W., Hu, D., \& Hao, J. (2017). International students' experiences in China: Does the planned reverse mobility work? International Journal of Educational Development, 61, 204-212.

Yamane, T. (1973). Statistics: An introductory analysis. Third edition. New York: Harper \& Row.

Yeh, C. J., \& Inose, M. (2003). International students' reported English 
fluency, social support satisfaction, and social connectedness as predictors of acculturative stress. Counselling Psychology Quarterly, 16(1), 15-28.

WEREDE TAREKE GEBREGERGIS is an assistant lecturer in the department of psychology, college of education, Eritrean Institute of Technology. Currently, he is pursuing his master degree in Applied Psychology at Central China Normal University, Wuhan, China. His research interests include cross-cultural studies, motivation, social and personality psychology, international students' adjustment issues, mental health issues and educational studies. E-mail: weredetarekeg@yahoo.com

HUANG FEI, PhD, is an Assistant Professor of psychology in the school of psychology, Central China Normal University, Wuhan, China. His research interest is social and personality psychology. E-mail: huangfei@mail.ccnu.edu.cn

JIANZHONG HONG, $\mathrm{PhD}$, is a professor of psychology in the school of psychology, Central China Normal University, Wuhan, China. His research interest include cross-cultural studies and applied psychology. E-mail:

jhong@mail.ccnu.edu.cn 